\title{
Digital watermarking method based on STFT histogram
}

Kotaro Sonoda (Nagasaki Univ, JP)

Aleksander Sek (Adam Mickiewicz Univ, PL) 


\section{Data Hiding}

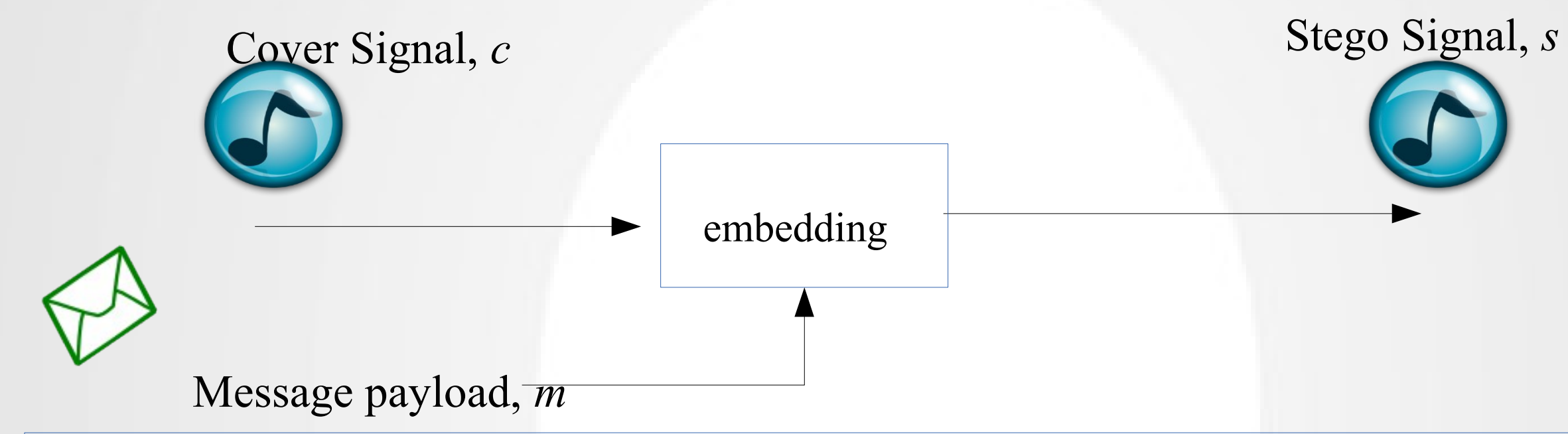

- Embedding is carried out in order to make the distortion of the Stego Signal from the cover signal under imperceptible.

- Watermarking model (main content is audio signal)

- User can't divide the message from Stego signal .

- ex) Copy Control, fingerprint

- Steganography (main content is message)

- User can't notice the existence of message.

- ex) Secret Communication, 


\section{Hidden message detection}

\section{Stego Signal, $s$}

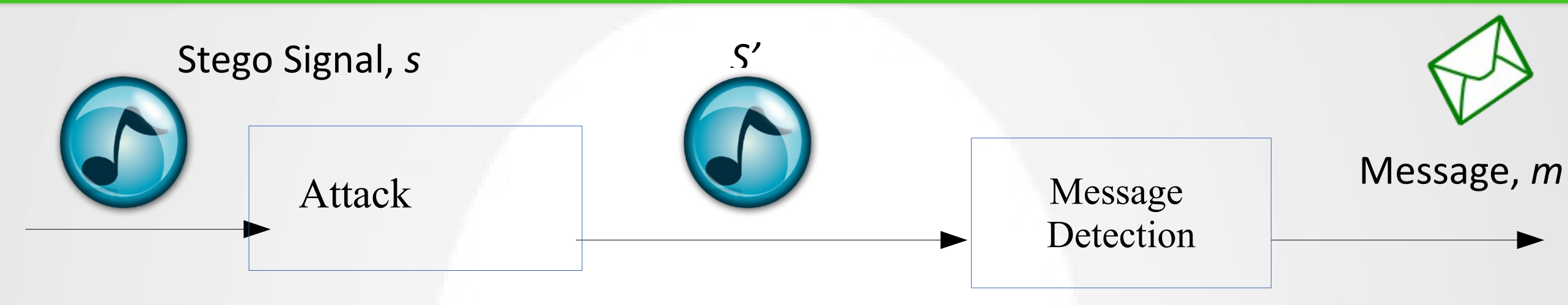

Noise, DA/AD,

Re-encoding, re-quantizization,

Mixing with other signal,

Transmitted through air,

Malicious attack by pirates

- Requirements

- As much message payload as possible $\rightarrow$ Capacity

- Resist against manipulations

- Inaudible distortion

$\rightarrow$ Robusteness

$\rightarrow$ Imperceptibility

- In generally, Robustness, Capacity, and Inaudibility are related each other.

- The most simple way to increase the robustness is changing the signal component gratefully to detect easily. $\rightarrow$ The audio quality might be deteriorated. 


\section{General aim for audio data hiding}

- Aim to detect the messages from a short segment (about $5 \mathrm{~s}$ ) cropped from anywhere of a piece of signal

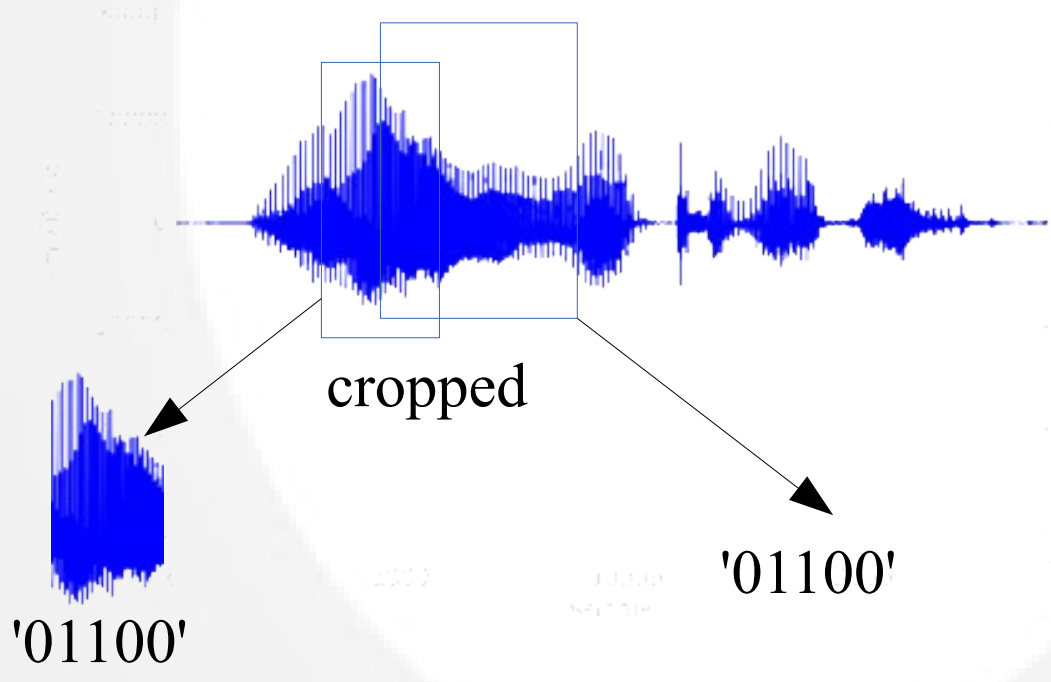




\section{Strategy on conventional methods}

- Embed messages into short segments, repeatedly all over the signal

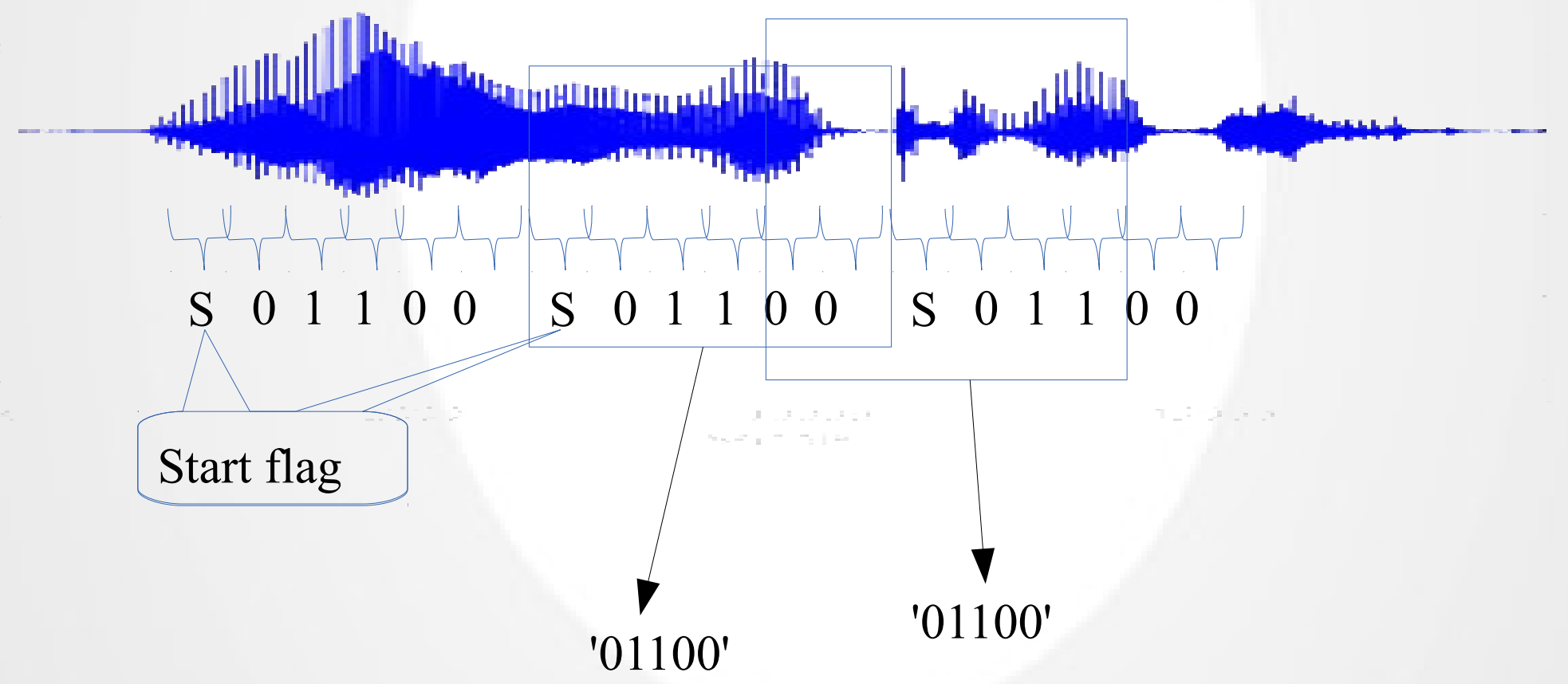

If the start point of analyzing segment is mismatched with one of the embedding sides, it is possible to fail to detect the messages. 


\section{Changing Histogram form of time variant powers}

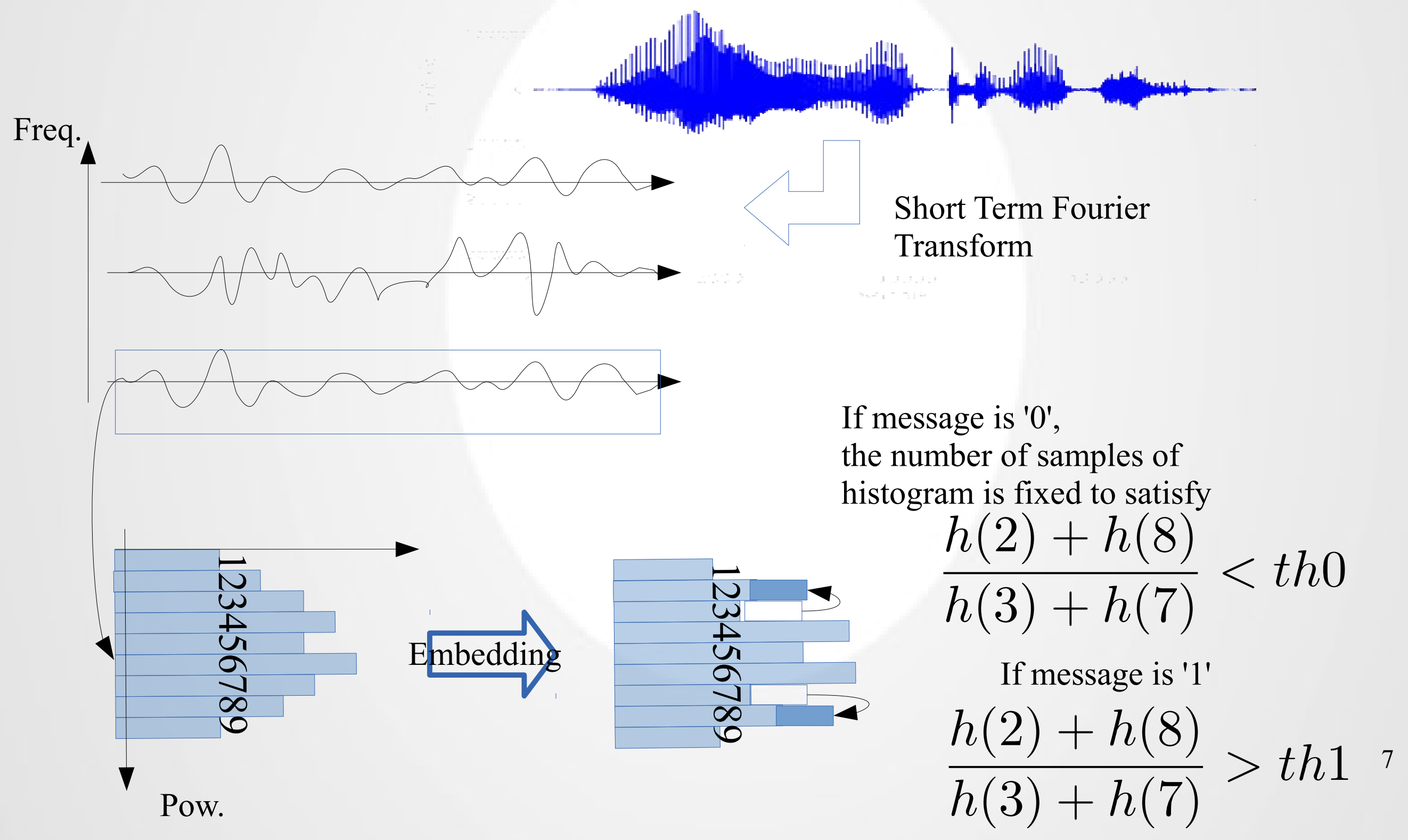




\section{Embedding (1)}
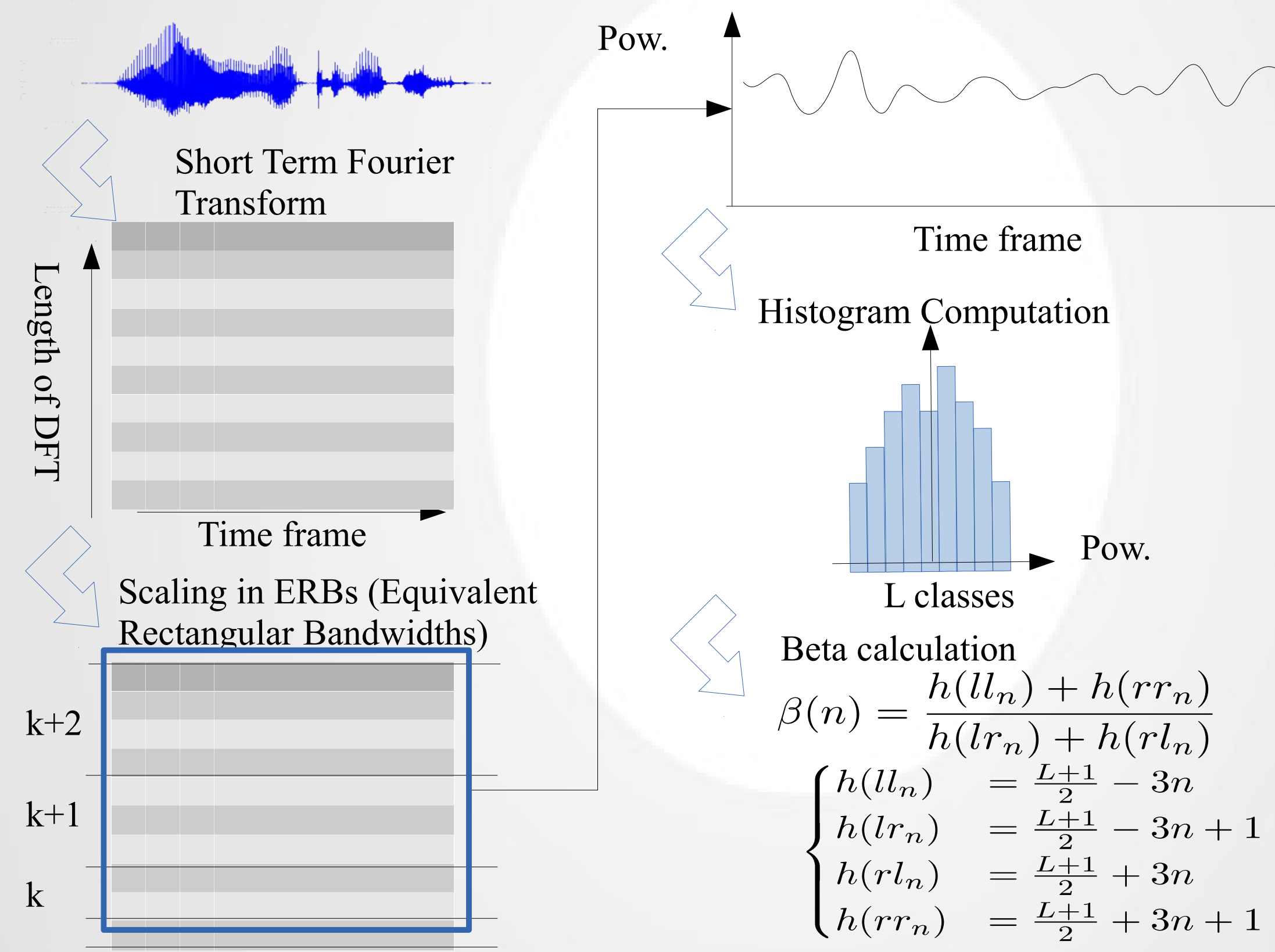


\section{Embedding (2)}

Pow.

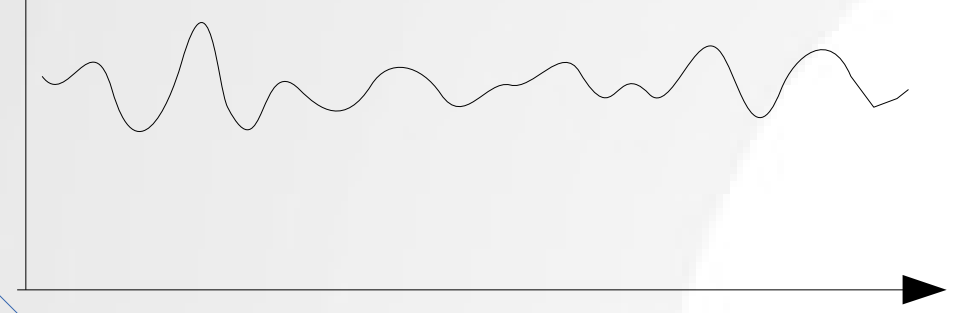

Time frame

Histogram Computation

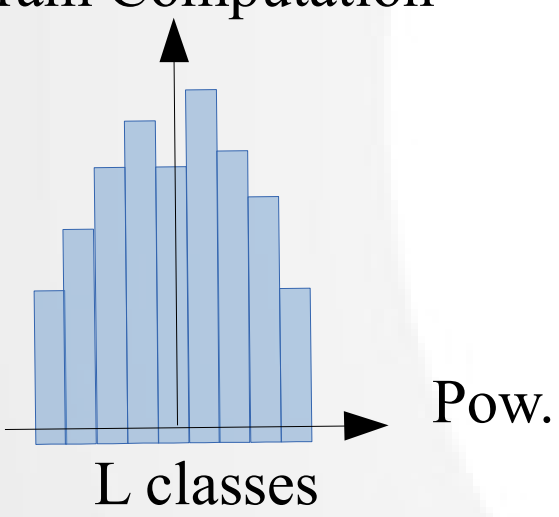

Beta calculation

$$
\begin{gathered}
\beta(n)=\frac{h\left(l l_{n}\right)+h\left(r r_{n}\right)}{h\left(l r_{n}\right)+h\left(r l_{n}\right)} \\
\left\{\begin{array}{l}
h\left(l l_{n}\right)=\frac{L+1}{2}-3 n \\
h\left(l r_{n}\right)=\frac{L+1}{2}-3 n+1 \\
h\left(r l_{n}\right)=\frac{L+1}{2}+3 n \\
h\left(r r_{n}\right)=\frac{L+1}{2}+3 n-1
\end{array}\right.
\end{gathered}
$$

Normalizing $\beta\left(n\right.$ to $\beta^{\prime}$ (ra)ccording to the following role defined for one bit of payload

$$
\left\{\begin{array}{l}
\beta^{\prime}(n)<t h_{\mathrm{O}} \\
\beta^{\prime}(n)>t h_{1} \Leftrightarrow w(n)=0
\end{array}\right.
$$

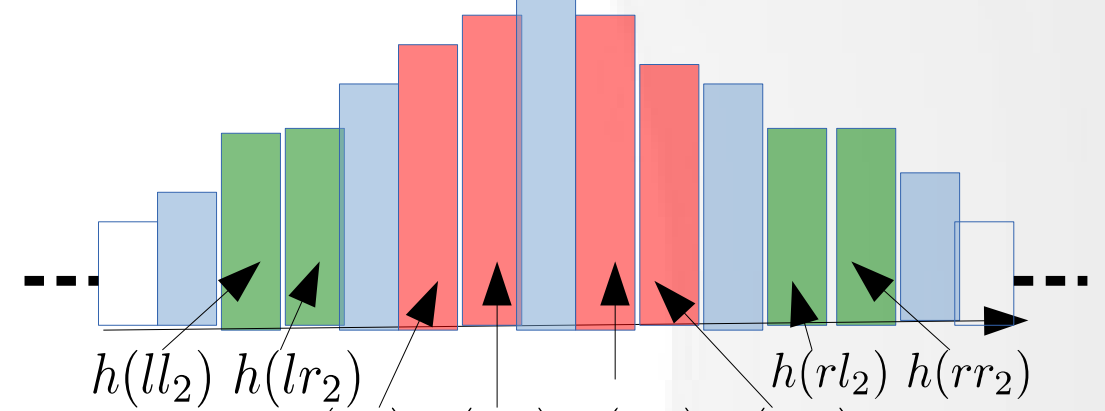

$h\left(l l_{2}\right) h\left(l r_{2}\right)$

$h\left(l l_{1}\right) h\left(l r_{1}\right) h\left(r l_{1}\right) h\left(r r_{1}\right)$
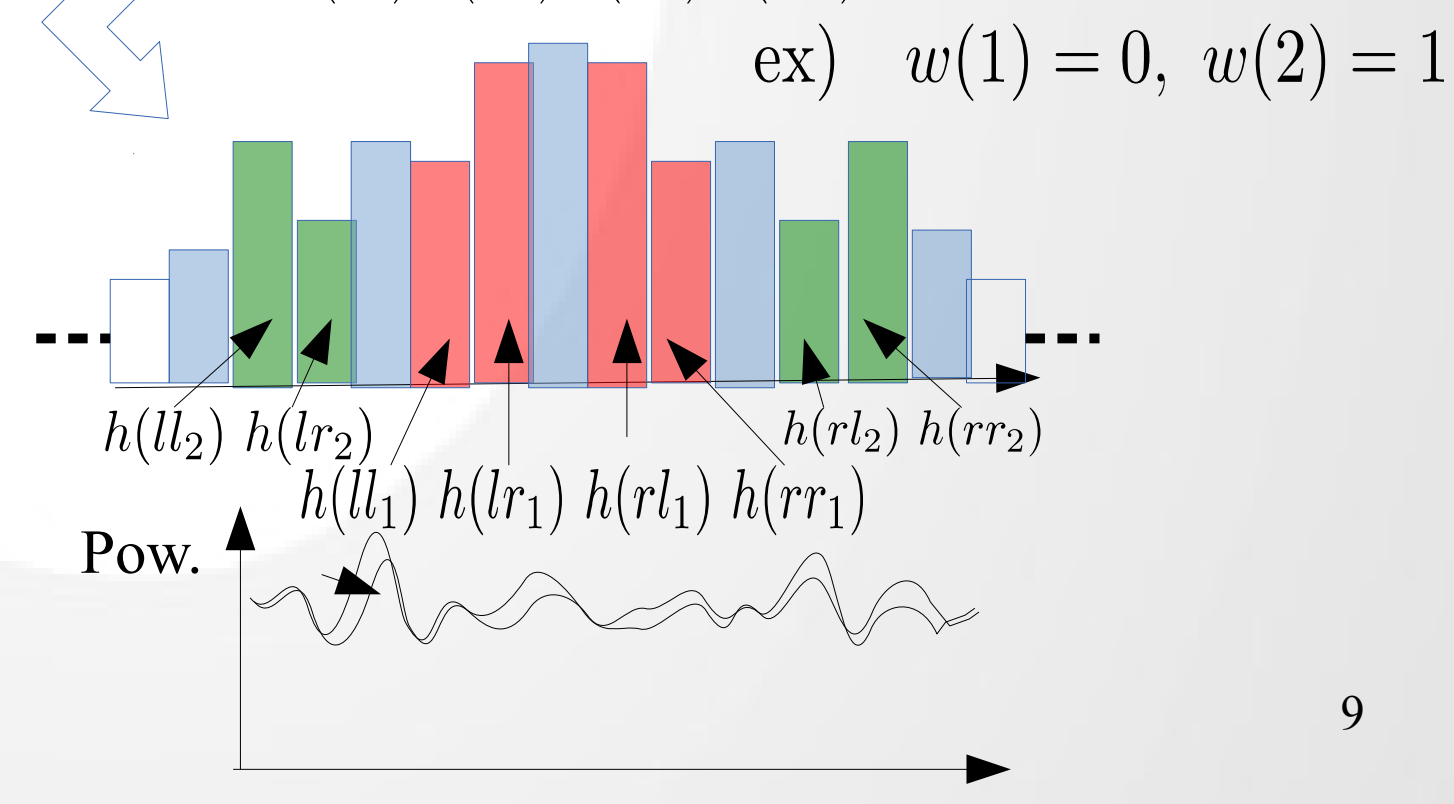

Time frame 


\section{Embedding (3)}

Normalizing $\beta$ ( $n$ to $\beta^{\prime}$ (ra)ccording to the following role defiend for one bit of payload

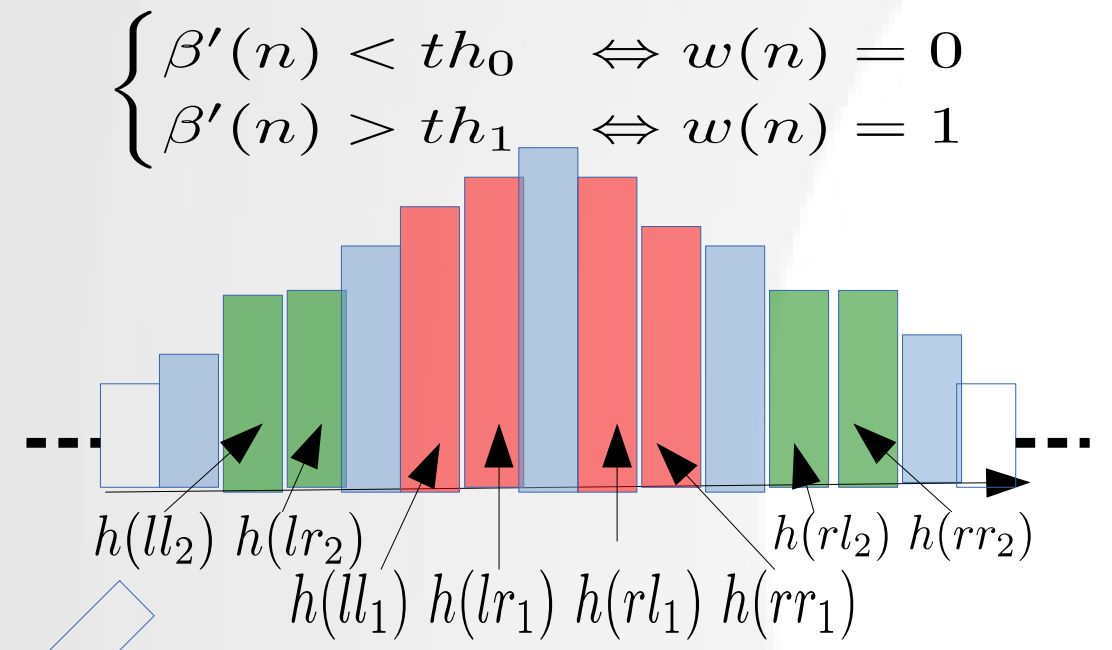

Replace coefficients with new value

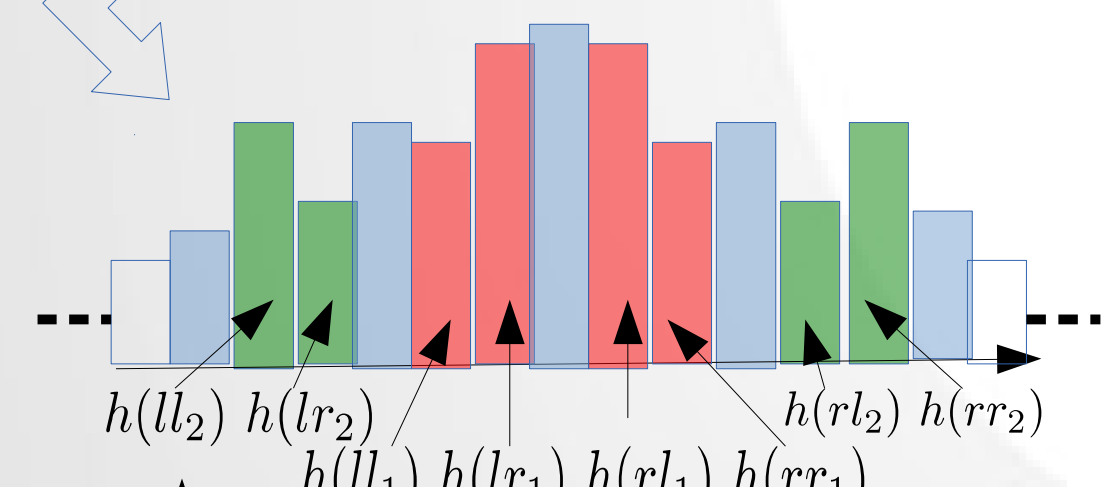

Pow. $\Delta \quad h\left(l l_{1}\right) h\left(l r_{1}\right) h\left(r l_{1}\right) h\left(r r_{1}\right)$

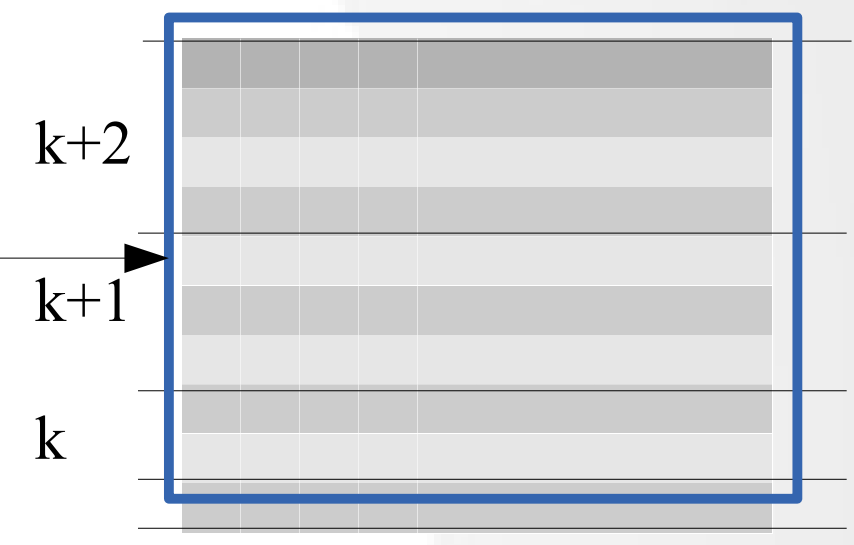

Inverse STFT

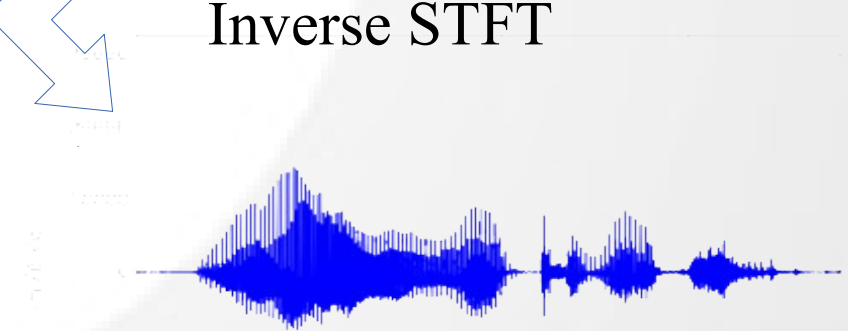

Time frame 


\section{Evaluation: Conditions}

\section{Imperceptibility}

Annoyance grade of Stego signal (S) from Cover signal (C).

\section{Robustness}

Bit error rate of extracted message (M') from Embedded message (M)

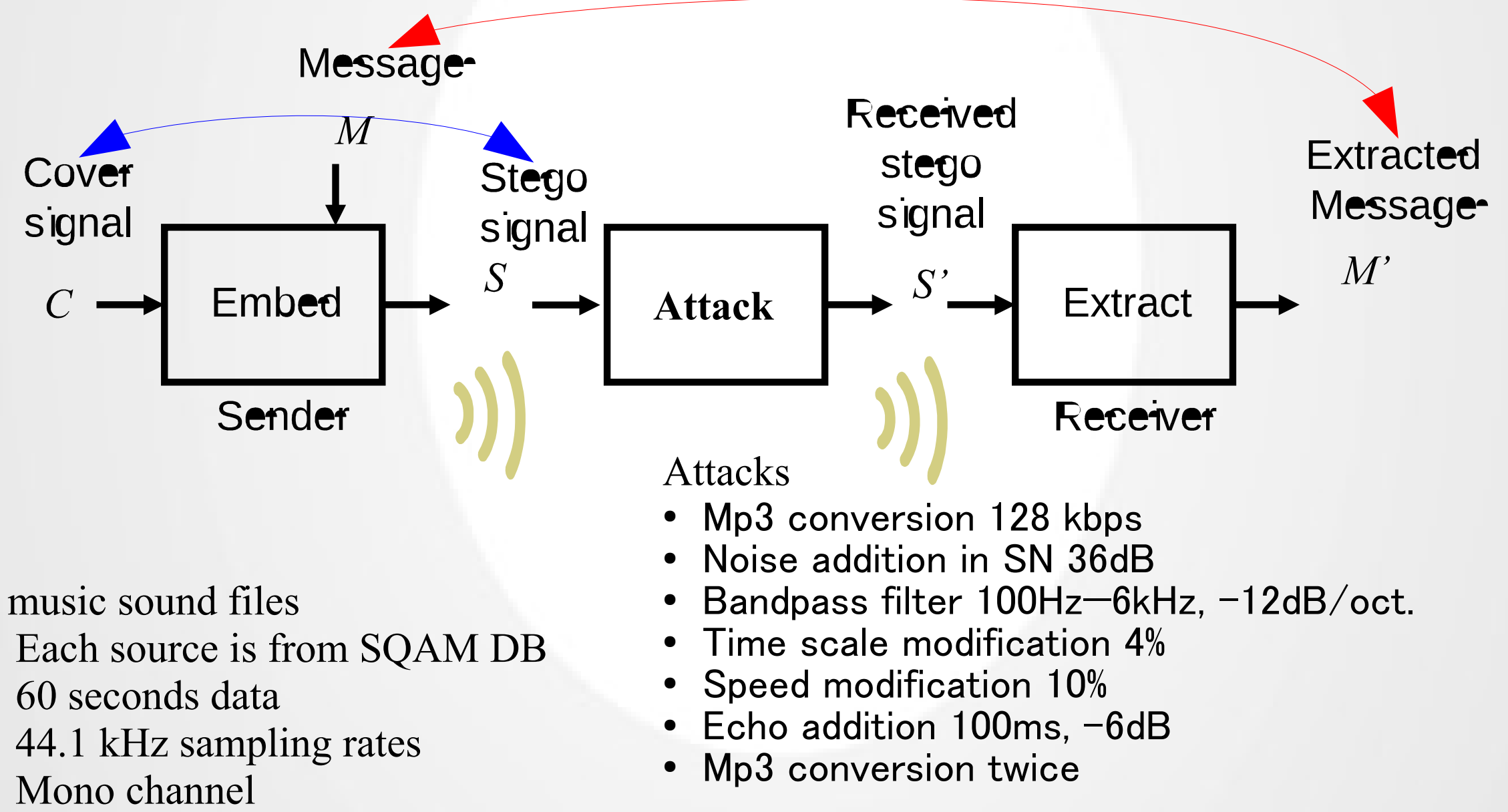




\section{Tested sound files}

EBU Sound Quality Assessment Material (SQAM)

Recordings for subjective tests

\section{Tested sound \# Ingredients}

27 Castanets

32 Triangles

35 Glockenspiel

40 Harpsichord

65 Orchestra

66 Wind ensemble

69 Pop music

70 Pop music

$+\quad 101$ Harmonic Complex tone 


\section{Watermarking conditions}

\begin{tabular}{|l|l|}
\hline parameter & setting \\
\hline Length of one segment & $25 \mathrm{~ms} \times 200$ frames $(5 \mathrm{~s})$ \\
\hline Number of bits of a payload $(\mathrm{N})$ & 4,8 \\
\hline Number of classes of histogram (L) & $6^{*} \mathrm{~N}+1(25,49)$ \\
\hline Thresholds & th0 $0=1 / 100$, th $1=100$ \\
\hline
\end{tabular}




\section{Robustness against MP3 conversion}
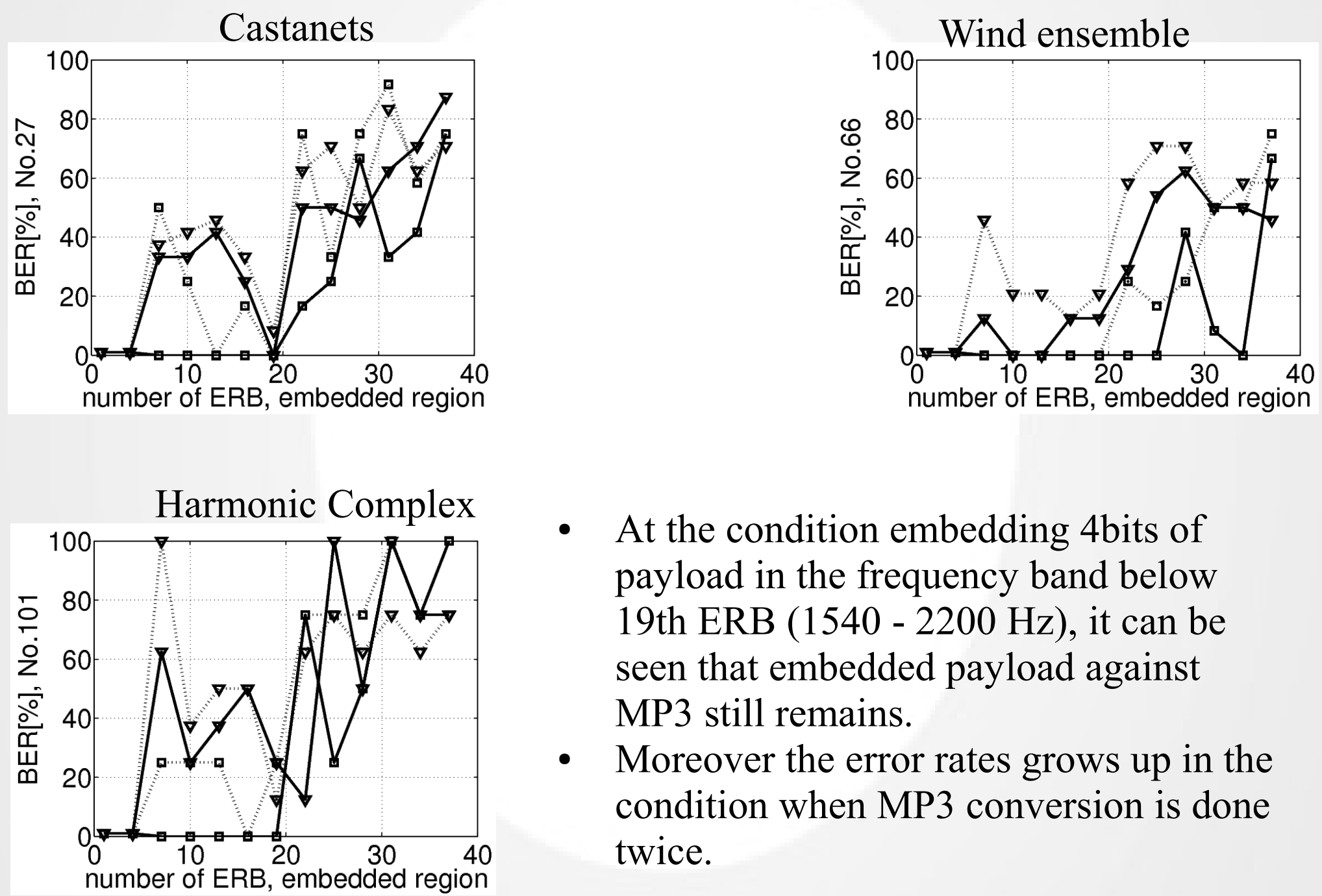

- At the condition embedding 4 bits of payload in the frequency band below 19th ERB (1540 - $2200 \mathrm{~Hz})$, it can be seen that embedded payload against MP3 still remains.

- Moreover the error rates grows up in the condition when MP3 conversion is done twice. 


\section{(Double-blind Triple-stimulus with Hidden Reference)}

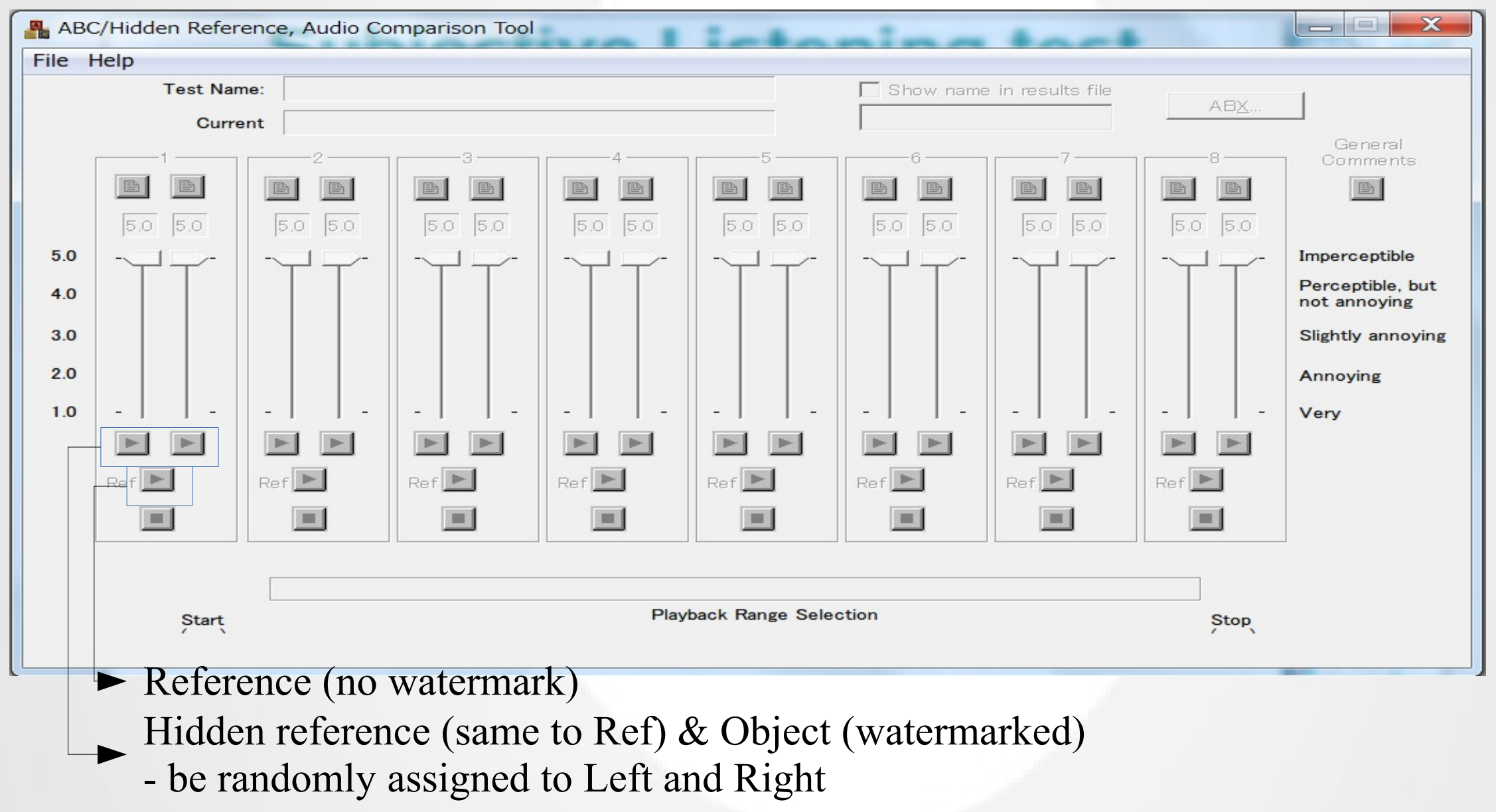

For each Left and Right botton's signal,

the subjects had to assess the audibility of potential distortions from Ref. Signal and grade from 1.0 (very annoying, totally distorted) up to 5.0 (Imperceptible, no distorted at all) 


\section{Results of Listening test ( 3 men \& 1 female)}
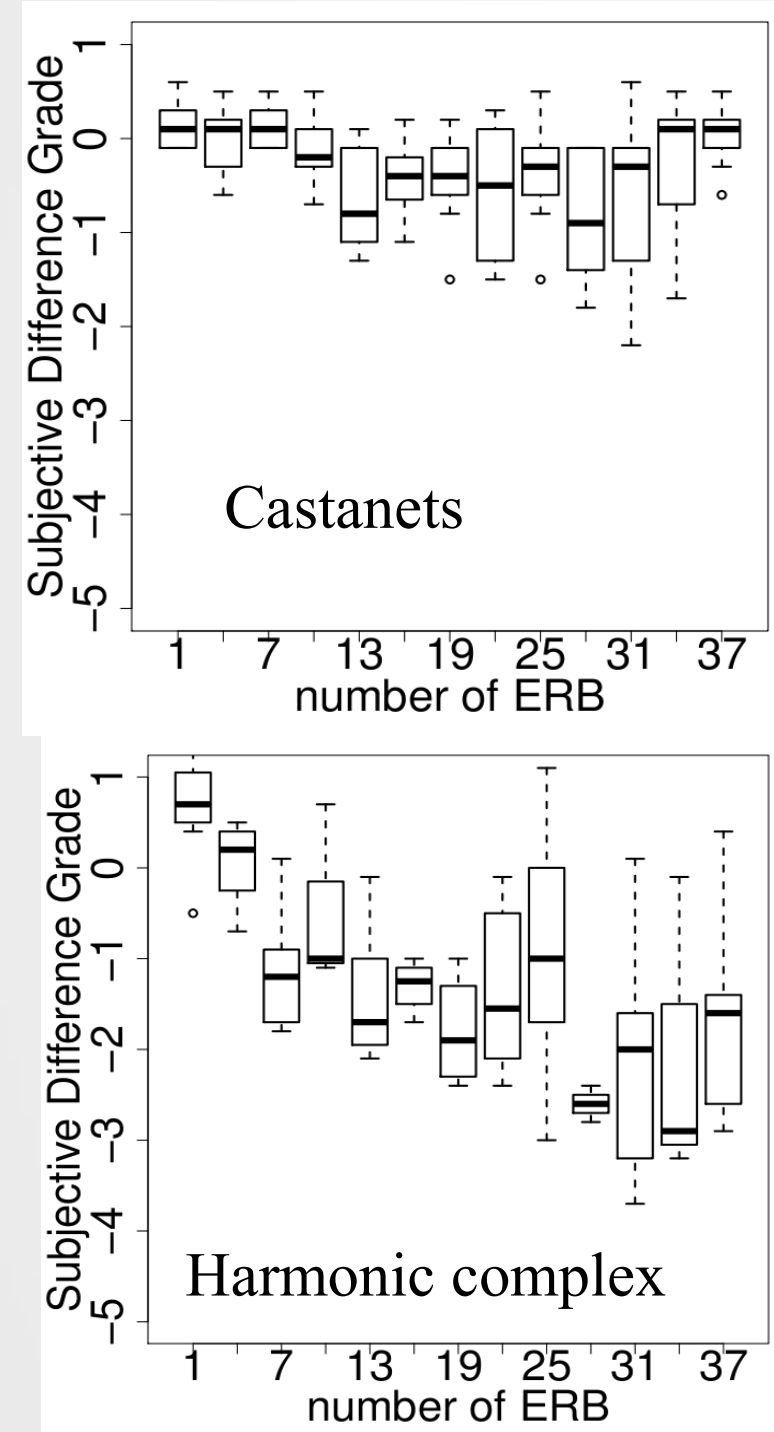

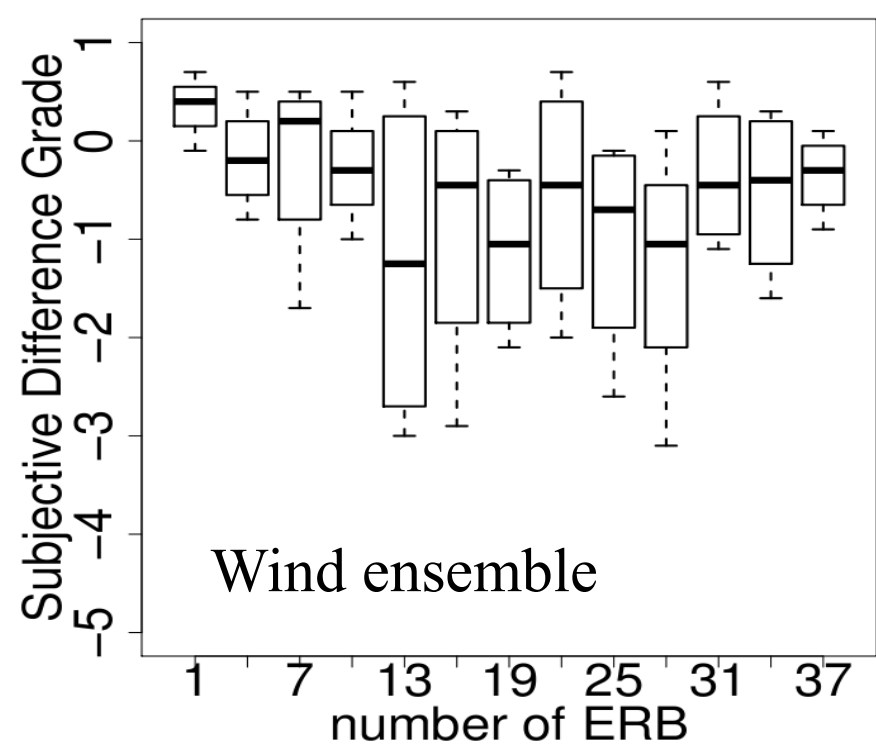

- Although the grades are varying between subjects, almost of the average SDGs are graded greater than -1 (Perceptible, but not annoying) for the instrumental signals or ensemble signals.

- In the case of the SDG for harmonic complex tone, the watermark was more annoying for most of the subjects when it was applied to a high frequency band.

$S D G=$ Grade $_{\text {Test }}-$ Grade $_{\text {Reference }}$ 


\section{Multiband embedding}

- In single band embedding, we can embed only 2 or 4 bits in 5 seconds. ( 0.4 or 0.8 bps!!)

- Embedding multi bits into multi-bands between $1^{\text {st }}$ and $20^{\text {th }}$ ERB region 


\section{Multiband embedding}

\section{(Embedding multi bits into multi-bands between $1^{\text {st }}$ and $20^{\text {th }}$ ERB region)}

\begin{tabular}{|c|c|c|c|c|c|c|}
\hline & \multicolumn{2}{|c|}{ castanets } & \multicolumn{2}{|c|}{ Wind ensemble } & \multicolumn{2}{|c|}{ Harmonic complex } \\
\hline & 27-mp3o & $27-\mathrm{mp} 3 \mathrm{t}$ & 66-mp3o & $66-\mathrm{mp} 3 \mathrm{t}$ & 101-mp3o & 101-mp3t \\
\hline 4 bits $\times 3$ (12 bits) & 0.00 & 2.78 & 0.00 & 0.00 & 0.00 & 0.00 \\
\hline 4 bits $\times 4$ (16 bits) & 0.00 & 10.42 & 0.00 & 0.00 & 0.00 & 6.25 \\
\hline 8 bits $\times 2$ (16 bits) & 8.33 & 37.50 & 8.33 & 22.92 & 0.00 & 12.50 \\
\hline 4 bits $\times 5$ (20 bits) & 3.33 & 16.67 & 0.00 & 3.33 & 0.00 & 15.00 \\
\hline 8 bits $\times 3$ (24 bits) & 16.67 & 43.06 & 6.94 & 20.83 & 25.00 & $\begin{array}{l}25.00 \\
(\mathrm{BER}[\%])\end{array}$ \\
\hline
\end{tabular}

- Results showed that in the multi-band case the proposed method can embed up to 20 bits (4 bps) without decrease in robustness against MP3. 


\section{Conclusion}

- Re-quantize powers of STFT coefficients, embedding in the form of histogram)

- Proposed method resists the MP3 compression attacks in the case of embedding payload below $2 \mathrm{kHz}$

- Proposed method doesn't produce audible distortions

- Multi-band embedding can embed up to 20 bits in 5 seconds without decrease in robustness against MP3 